\title{
YOGURT NATURAL A PARTIR DE LECHE CON SUERO LÁCTEO A ESCALA DE LABORATORIO, UNIVERSIDAD NACIONAL DE INGENIERÍA, SEDE REGIONAL DEL NORTE, ESTELÍ NICARAGUA
}

\section{NATURAL YOGUR OBTAINED FROM MILK WITH DAIRY SERUM A LABORATORY SCALE, NATIONAL UNIVERSITY OF ENGINEERING, NORTH REGIONAL HEADQUARTERS, ESTELÍ NICARAGUA}

\author{
Mariliana Videa Bustillo' \\ Mabel Vanessa Videa Zelaya²
}

(Recibido/received: 27-Noviembre-2019; aceptado/accepted: 13-Diciembre-2019)

RESUMEN: La investigación tuvo como finalidad la elaboración de una bebida fermentada (yogurt) resultante de una mezcla entre suero lácteo y leche entera, utilizando tres formulaciones con diferentes proporciones de suero y leche entera 30/70, 50/50 y 70/30, respectivamente. Cumpliendo con las propiedades organolépticas óptimas, buscando la reducción en los costos de producción y a la vez la reutilización del suero lácteo por ser un agente contaminante del ambiente. Para iniciar la elaboración del yogurt, se caracterizó la materia prima utilizando leche fresca entera de vaca y suero fresco del procesamiento de queso, ambas sustancias (leche y suero) se sometieron a análisis físico químico (temperatura, densidad y acidez titulable). Se prepararon los tres tipos de formulaciones de yogurt y se determinó por análisis sensorial que la fórmula que contenía 70\% leche y $30 \%$ suero, presentó mejores características organolépticas. Con la formulación seleccionada, se realizaron los cálculos de rendimiento productivo y los balances de masa, encontrando que el rendimiento en relación a $3.68 \mathrm{~kg}$ de mezcla leche-suero, equivalente al 100\% fue de $2.11 \mathrm{~kg}$ de yogurt, es decir 54\%, siendo este el rendimiento de la producción. A la vez, se calcularon los costos generales para producir 100 envases de yogurt de $250 \mathrm{ml}$ cada uno y se encontró que el costo de producir los 100 envases fue de $C \$ 1,777.27$, dando un costo por unidad de C\$17.77.

PALABRAS CLAVE: fermentación; formulación; análisis sensorial; lactosuero.

\footnotetext{
${ }_{1}$ Coordinadora de Ingeniería Agroindustrial UNI RUACS. mariliana.videa@norte.uni.edu.ni

2 Graduada de Ingeniería Agroindustrial UNI RUACS, Nicaragua.
} 
ABSTRACT: The purpose of the research was to prepare a fermented beverage (yogurt) resulting from a mixture between whey and whole milk, using three formulations with different proportions of whey and whole milk 30/70, 50/50 and 70/30, respectively. Complying with the optimal organoleptic properties, seeking the reduction in production costs and at the same time the reuse of whey as a pollutant of the environment. To start the yogurt production, the raw material was characterized using fresh whole cow's milk and fresh whey from the cheese processing, both substances (milk and whey) were subjected to chemical physical analysis (temperature, density and titratable acidity). The three types of yogurt formulations were prepared and it was determined by sensory analysis that the formula containing $70 \%$ milk and $30 \%$ whey presented better organoleptic characteristics. With the selected formulation, calculations of productive performance and mass balances were performed, it was found that the yield in relation to $3.68 \mathrm{~kg}$ of milk-whey mixture, equivalent to $100 \%$ was $2.11 \mathrm{~kg}$ of yogurt, that is $54 \%$, this being the production yield. At the same time, the general costs to produce 100 yogurt containers of $250 \mathrm{ml}$ each were calculated and the cost of producing the 100 containers was found to be $C \$ 1,777.27$, giving a cost per unit of $\mathrm{C} \$ 17.77$.

KEYWORDS: fermentation; formulation; sensorial analysis; whey.

\section{INTRODUCCIÓN}

La industria láctea es uno de los sectores más importantes de la economía de países industrializados y en desarrollo. Aproximadamente el $90 \%$ del total de la leche utilizada en la industria quesera es eliminada como lactosuero, el cual retiene cerca del $55 \%$ del total de ingredientes de la leche como la lactosa, proteínas solubles, lípidos y sales minerales (Parra, 2009).

El lactosuero es un subproducto que se obtiene de la elaboración de queso el cual se caracteriza por poseer un color amarillo-verdoso y la forma opalescente, además de un alto valor nutritivo, esto por la presencia de proteínas disueltas en el mismo con alto valor biológico (entre la que se destacan la $\alpha$-lacto-albúmina y la $\beta$-lacto- globulina); las vitaminas del complejo B, y minerales tales como el calcio y el fósforo (Parra, 2009).

A nivel global, la utilización de suero lácteo está muy difundida por su valor nutritivo y menor costo, siendo aprovechado como materia prima para la elaboración de otros productos de consumo humano entre ellos está el yogurt (Montesdeoca, Benítez, \& Guevara Raúl, 2017). Por lo antes mencionado, en esta investigación se propuso la obtención de yogurt a partir de tres formulaciones de suero y leche entera en proporciones de 30/70, 50/50 y 70/30, y se comprobó por medio de análisis sensorial la mejor formulación, determinándose los respectivos costos de producción. 


\section{METODOLOGÍA}

Este estudio se llevó a cabo en el laboratorio de agroindustria de la Universidad Nacional de Ingeniería, Recinto Universitario Augusto C. Sandino, Sede Regional del Norte, entrada la Tunosa ubicada en la ciudad de Estelí- Nicaragua.

Para alcanzar los objetivos de la investigación primero se caracterizó la materia prima, la leche fresca entera de vaca y el suero fresco proveniente del procesamiento del queso. Para esto se tomaron en cuenta las propiedades fisicoquímicas de ambas sustancias.

- Medición de pH: Para ello se utilizó un pH metro digital y para la medición de temperatura un termómetro infrarrojo con un rango de $-18^{\circ} \mathrm{C}$ a $400^{\circ} \mathrm{C}$.

- Acidez titulable: Según el documento Métodos para el análisis fisicoquímico de la leche y derivados lácteos, la acidez titulable se realiza mediante el método volumétrico que consiste en una titulación con solución valorada de hidróxido de sodio, frente a fenolftaleína como indicador hasta un color rosado (Artica, 2014).

- Densidad: Según el procedimiento para determinar la densidad relativa la prueba de densidad se realiza haciendo uso del lactodensímetro.

Para determinar la fórmula de leche y suero para la obtención de yogurt, se elaboró el yogurt con las tres formulaciones propuestas $(70 / 30,50 / 50$ y 30/70) y se sometieron a análisis sensorial con un panel experto de 15 jueces en una prueba orientada al producto (Watts, G.L, \& Jeffrey, 1992). En esta prueba se evaluaron los parámetros color, olor, sabor, uniformidad, cremosidad y acidez de las muestras de yogurt en las diferentes formulaciones leche-suero, utilizando una escala Likert de 1 a 5.

Para el análisis de los datos se hizo utilizando el software InfoStat (Di Rienzo J.A., 2016) con análisis de la varianza (ANDEVA) con un diseño de bloques completos al azar, donde el bloque es la persona que evaluó las muestras.

Una vez seleccionada la muestra mejor evaluada de las tres formulaciones propuestas, se sometió a una comparación con yogurt 100\% leche, para esto se realizó un análisis sensorial con 50 panelistas no entrenados en una prueba orientada al consumidor (Watts, G.L, \& Jeffrey, 1992). Para este análisis, se utilizó la prueba T de Student para muestras independientes.

Para la realización del producto se llenó una tabla de recolección de datos de los volúmenes y pesos iniciales y finales por cada etapa del proceso tanto para la materia prima como para los insumos y aditivos, esto con el propósito de calcular el rendimiento de masa que se realizó a partir de los porcentajes de las entradas y salidas tanto de materia prima como de producto terminado. Para esto se utilizó la ecuación general de balance de materia y energía planteada por (Murphy, 2007).

El Higo Revista Científica / Volumen 09.No.01, pp. 59-68 / Diciembre 2019 
Ecuación 1. Ecuación de balance de materia.

$$
[\text { Entrada }]+[\text { Generación }]-[\text { Salida }]-[\text { Consumo }]=[\text { Acumulación }]
$$

Utilizando diagramas de entradas -salidas como el que se muestra en la figura1.

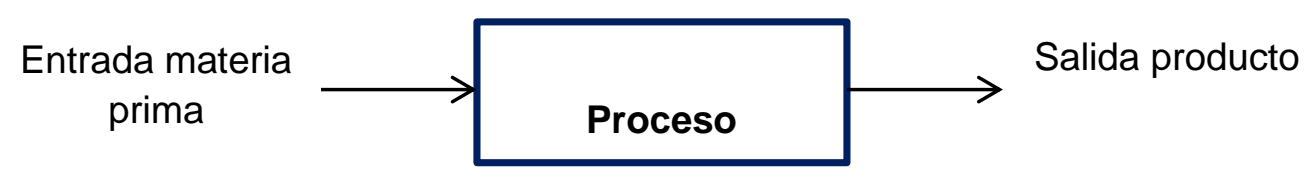

Figura1. Diagrama de entradas y salidas.

Un solo bloque representa todas las operaciones físicas y químicas involucradas en el proceso. Las líneas con flechas representan el movimiento de materiales que entraron y salieron del proceso (Murphy, 2007).

\section{RESULTADOS Y DISCUSIÓN}

Se caracterizó la leche y el suero utilizados en la elaboración del yogurt en las tres formulaciones planteadas y los resultados se muestran en la tabla 1.

Tabla 1. Resultados de la caracterización físico química de la materia prima

\begin{tabular}{lcc}
\hline \multicolumn{1}{c}{ Parámetro de } & \multicolumn{2}{c}{ Resultados } \\
\cline { 2 - 3 } \multicolumn{1}{c}{ referencia } & Leche & Suero \\
\hline pH & 6.2 & 6.0 \\
Acidez titulable & 0.17 & 0.073 \\
Densidad & $1.28 \mathrm{~kg} / \mathrm{m}^{3}$ & $1.17 \mathrm{~kg} / \mathrm{m}^{3}$ \\
\hline
\end{tabular}

La leche debe poseer un pH de entre 6.0 - 6.7 (Artica, 2014), los resultados de acidez corresponden a leche fresca. Así mismo los datos obtenidos del pH del suero, indican que este fue obtenido de leche fresca y que el mismo es suero fresco. En el caso de la acidez, los valores aptos para leche son de $0.14-0.18 \%$ de acidez (Negri, 2017), resultando así que la leche y el suero utilizados no presentan problemas en cuanto a la acidez.

De las tres fórmulas se realizó una selección previa mediante degustaciones donde se evaluaron las características organolépticas de cada una y así determinar la mejor muestra, con la participación de 15 jueces expertos. De forma general se presentan los resultados de esta evaluación en la figura 2. 


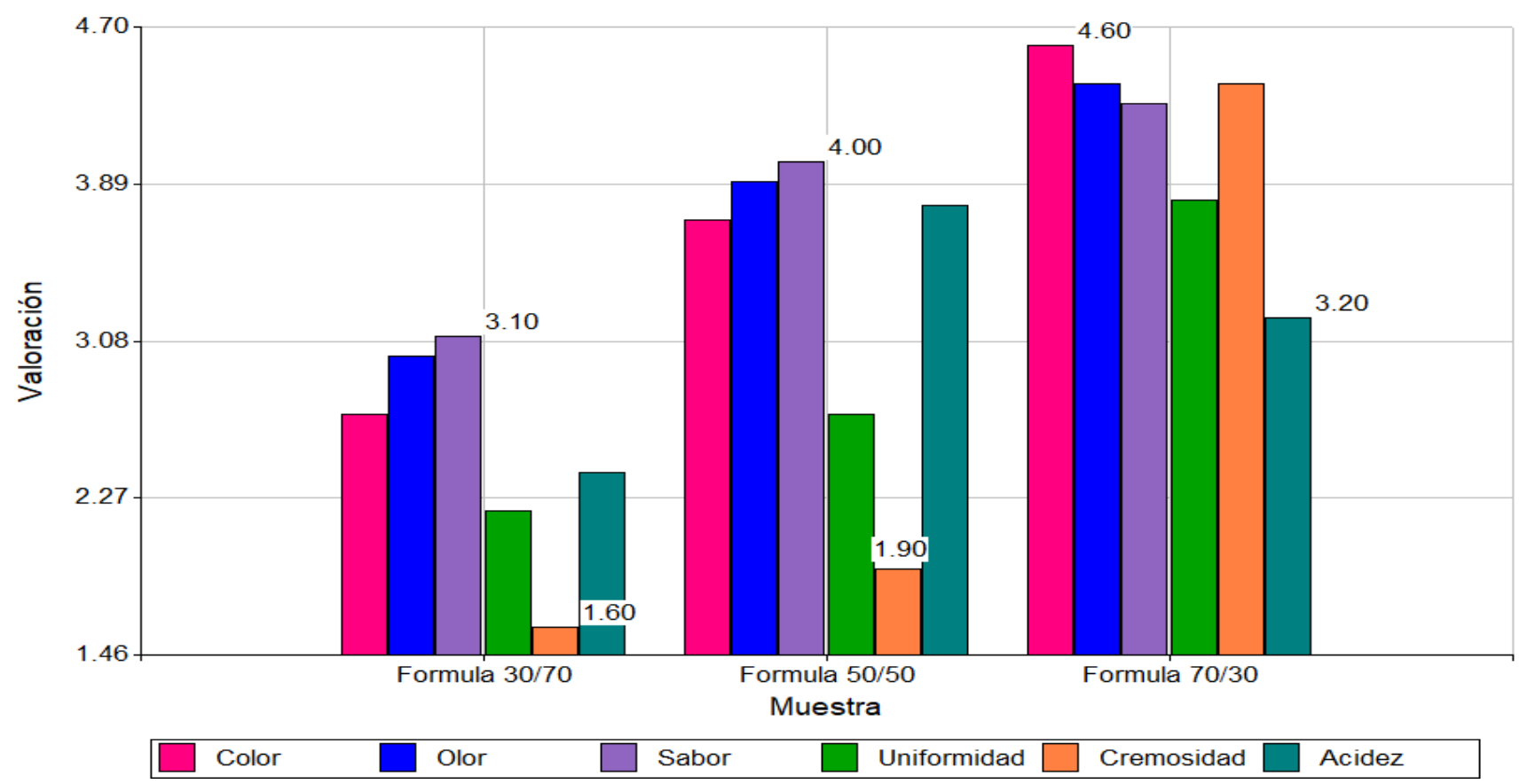

Figura 2. Valoraciones dadas por los jueces expertos a las muestras de yogurt.

Para la fórmula $30 / 70$ (\% de leche/\% de suero) el criterio mejor evaluado fue el sabor con un promedio de 3.10 que según la escala planteada indica que la muestra de yogurt no les disgustó, pero tampoco les gustó. El criterio que tuvo menor valoración para esta muestra fue la cremosidad, indicando que el yogurt estaba "ralo", dato que concuerda con Miranda, Fonseca, Ponce y Cedeño (2014) quienes elaboraron yogurt a base de suero e indican que la viscosidad del mismo es poca. Para la fórmula 70/30, todas las valoraciones a las características organolépticas están por encima del valor 3 , es decir más cerca de la valoración ideal.

Se analizaron los datos por medio de un análisis de varianza con un diseño de bloques completos al azar, los resultados se muestran en la tabla 2.

Tabla 2. Análisis de varianza de los atributos evaluados en las tres formulaciones de yogurt

\begin{tabular}{lcr}
\hline \multicolumn{1}{c}{ Variable/p-valor } & Juez & \multicolumn{1}{c}{ Muestra } \\
\hline Color & 0.1765 & 0.007 \\
Olor & 0.9120 & $\mathbf{0 . 0 6 9 9}$ \\
Sabor & 0.4968 & 0.0404 \\
Uniformidad & 0.0732 & 0.0023 \\
Cremosidad & 0.7220 & $<0.0001$ \\
Acidez & 0.7933 & 0.0320 \\
\hline
\end{tabular}


Los panelistas si encontraron diferencias significativas entre las muestras, excepto para el olor, al que consideraron igual para las tres fórmulas. En la tabla 3. Se presenta cuáles son las muestras diferentes en la prueba de Duncan.

Medias seguidas de letra mayúscula igual en la misma columna no difieren entre sí al nivel de significancia del $5 \%$ por la prueba de Duncan.

Tabla 3. Separación de promedios para los atributos evaluados en las tres formulaciones

\begin{tabular}{cccccc}
\hline $\begin{array}{c}\text { Formulación } \\
\text { / Variables }\end{array}$ & Color & Acidez & Sabor & Uniformidad & Cremosidad \\
\hline $\mathbf{3 0 / 7 0}$ & $2.70^{\mathrm{A}}$ & $2.22^{\mathrm{A}}$ & $3.10^{\mathrm{A}}$ & $2.20^{\mathrm{A}}$ & $1.60^{\mathrm{A}}$ \\
$\mathbf{5 0 / 5 0}$ & $3.70^{\mathrm{B}}$ & $3.22^{\mathrm{AB}}$ & $4.00^{\mathrm{AB}}$ & $2.70^{\mathrm{A}}$ & $1.90^{\mathrm{A}}$ \\
$\mathbf{7 0 / 3 0}$ & $4.60^{\mathrm{C}}$ & $3.78^{\mathrm{B}}$ & $4.30^{\mathrm{B}}$ & $3.80^{\mathrm{B}}$ & $4.40^{\mathrm{B}}$ \\
\hline
\end{tabular}

Como se muestra en la tabla 3 la formulación que resulta diferente en todos los casos es la $70 / 30$ con medias más altas. En el caso del color las tres formulaciones resultaron diferentes pero la fórmula 70/30 fue mejor evaluada.

Después de los análisis realizados se encontró que la fórmula mejor evaluada y que resultó diferente al resto fue la fórmula $70 \%$ leche y $30 \%$ suero, ésta fue sometida al análisis sensorial final con una muestra testigo de yogurt $100 \%$ leche, para ello se contó con 50 panelistas, los resultados se muestran en la figura 3.

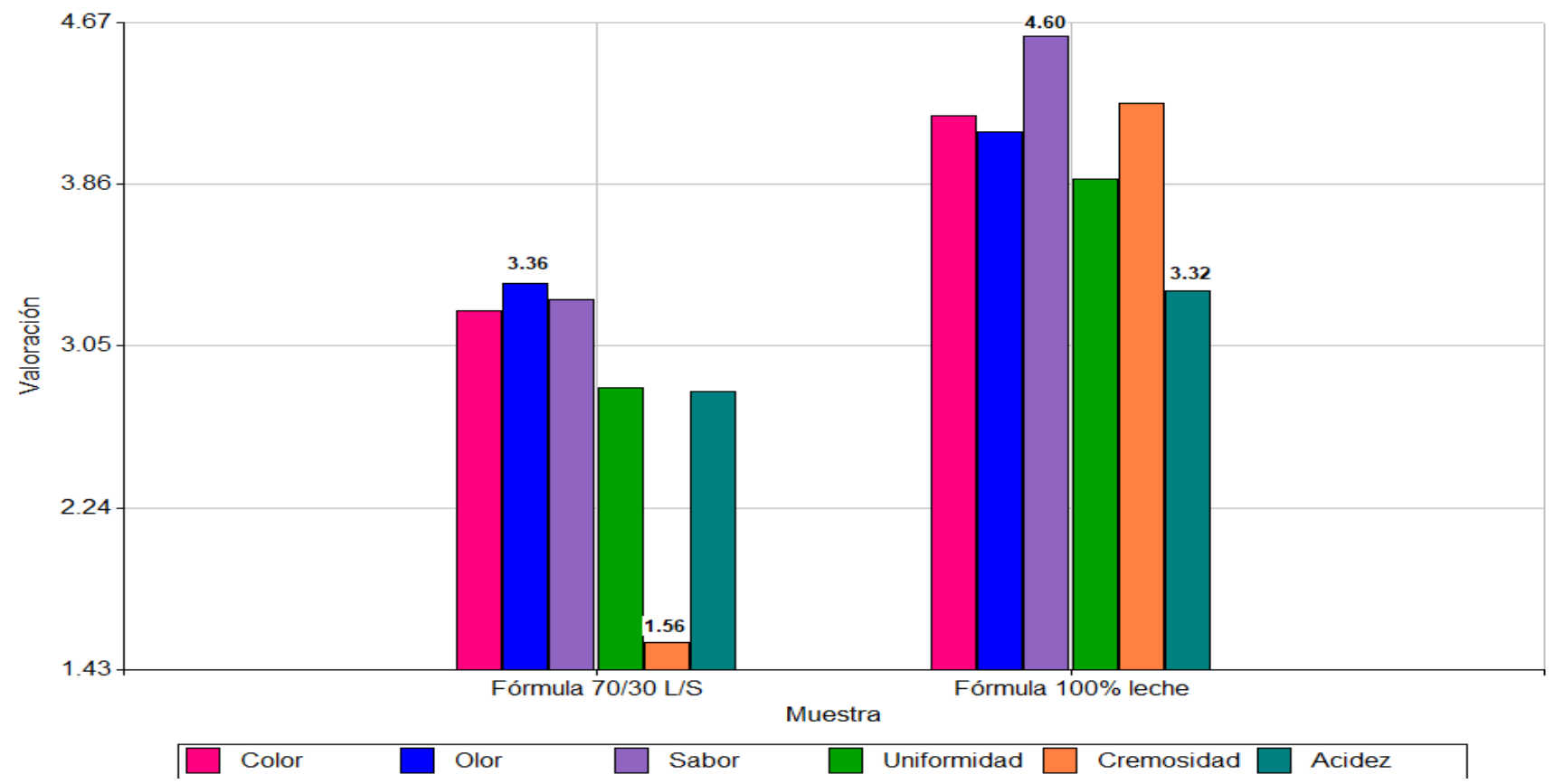

El Higo Revista Científica / Volumen 09.No.01, pp. 59-68 / Diciembre 2019 
Figura 3. Valoración de los jueces no entrenados a las muestras de yogurt. Es notable que las valoraciones más altas fueron dadas a la muestra de yogurt elaborada con $100 \%$ leche, teniendo mayor puntaje el sabor que indica que a los jueces les gustó mucho este parámetro, el valor menor fue dado a la acidez lo que muestra que el yogurt no se consideró ni muy ácido ni muy dulce.

Se analizaron los resultados de las evaluaciones sensoriales para comparar y determinar si existen diferencias significativas entre la fórmula propuesta y el testigo, estos se presentan en la tabla 4.

Tabla 4. Resultado de la prueba t para muestras independientes.

\begin{tabular}{cccc}
\hline Variable & Fórmula & Media & p valor \\
\hline Color & $100 \%$ leche & 4.2 & $<0.0001$ \\
& $70 / 30$ & 3.22 & \\
Olor & $100 \%$ leche & 4.12 & 0.0002 \\
& $70 / 30$ & 3.36 & \\
Sabor & $100 \%$ leche & 4.60 & $<0.0001$ \\
& $70 / 30$ & 3.28 & \\
Uniformidad & $100 \%$ leche & 3.88 & $<0.0001$ \\
& $70 / 30$ & 2.84 & \\
Cremosidad & $100 \%$ leche & 4.26 & $<0.0001$ \\
& $70 / 30$ & 1.56 & \\
Acidez & $100 \%$ leche & 3.32 & 0.0045 \\
& $70 / 30$ & 2.82 & \\
\hline
\end{tabular}

Todos los valores $\mathrm{p}$ son significativamente diferentes, es decir que los panelistas si encontraron diferencias en todos los parámetros evaluados para las muestras de yogurt. Y en este sentido, se observó los valores promedios de cada variable, la fórmula 70/30 que es la propuesta, obtuvo los valores menores. Los valores $\mathrm{p}$ más bajos son los del color, sabor, cremosidad y uniformidad, considerándose como los parámetros que encontraron con mayor diferencia.

Se realizaron los cálculos de rendimiento de la formulación propuesta, en la figura 4 se presenta el diagrama de flujo de la producción de yogurt utilizando leche y suero en la proporción seleccionada (70/30), con las entradas y salidas del proceso, finalmente el rendimiento en la obtención de este producto. 


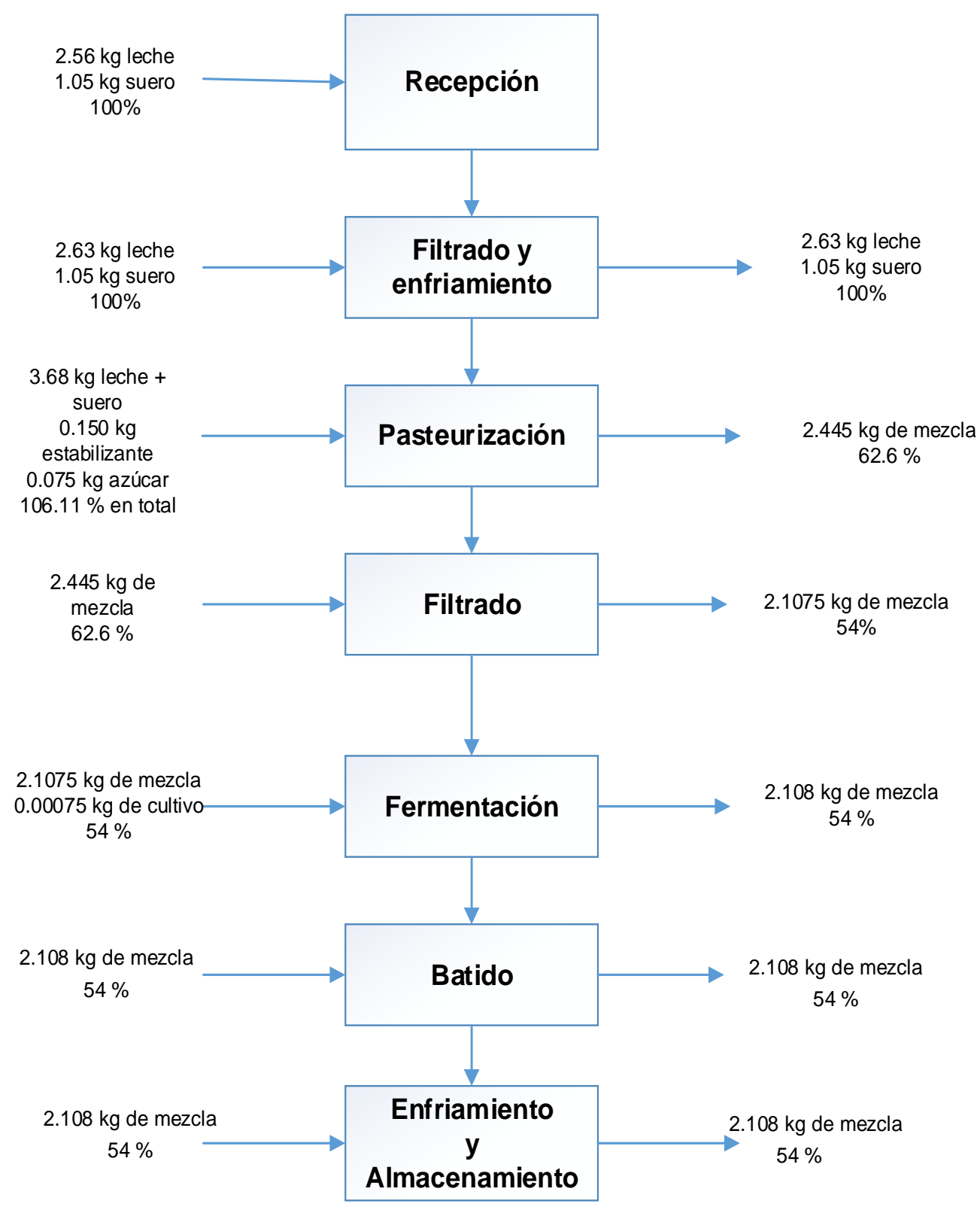

Figura 4. Diagrama de flujo para la producción de yogurt.

El rendimiento del proceso fue de $54 \%$, en yogurt elaborado a partir de $100 \%$ leche de vaca considerando un rendimiento bajo de acuerdo a lo planteado por Román (2005), donde obtuvo un rendimiento de producción del 93.87\%.

En estas condiciones un envase de yogurt de $250 \mathrm{ml}$ tiene un costo de producción de $\mathrm{C} \$$ 17.77 por unidad. Precio que resulta ser favorable tomando en cuenta que los precios de yogurt comercial de marcas Parmalat y La Perfecta oscilan entre $C \$ 19.50$ y $C \$ 20.00$, marca Eskimo, C\$27, todas estas en presentaciones de envases de $235 \mathrm{ml}$. Es importante recalcar que el costo calculado, es un costo general y no considera los costos de operación

El Higo Revista Científica / Volumen 09.No.01, pp. 59-68 / Diciembre 2019 
y otros costos fijos y variables, por tanto, el precio aumentará, sin embargo, puede competir con los precios de otras marcas comerciales como ya se mencionó.

\section{CONCLUSIÓN}

La materia prima utilizada fue leche fresca entera de vaca y suero fresco del procesamiento de queso, ambos provenientes de Lácteos Mendoza, donde se encargan de cumplir con todos los requisitos de calidad en el manejo de lácteos. A ambos líquidos (leche y suero) se les realizó análisis físico químico, indicando los resultados que se trató de leche y suero frescos aptos para la producción de yogurt.

Se formularon 3 tipos de mezclas para la elaboración de yogurt con respecto a las cantidades de leche y suero a utilizarse, la primera fue $70 \%$ leche y $30 \%$ suero, la segunda $50 \%$ para ambas sustancias, la tercera $70 \%$ suero y $30 \%$ leche.

Los datos obtenidos en las hojas de recolección para análisis sensorial fueron evaluados por medio de un análisis de varianza bifactorial sin interacción que es equivalente al modelo matemático de un diseño de bloques completos al azar, donde los bloques estuvieron representados por los jueces del panel sensorial. Con el resultado de este análisis se determinó que la fórmula con la que se elaboraría el yogurt sería la que contenía $70 \%$ leche y $30 \%$ suero, ya que presentó diferencia significativa $(p<0.05)$.

Con la formulación seleccionada, se realizaron los cálculos de rendimiento productivo y los balances de masa, se encontró que el rendimiento es de $54 \%$. Se calcularon los costos generales para producir 100 envases de yogurt de $250 \mathrm{ml}$ cada uno y se encontró que es de $C \$ 1,777.27$, dando un costo por unidad de $C \$ 17.77$.

Por último, se realizó una comparación en precios con respecto a las marcas comerciales, resultando que el producto elaborado obtiene un buen precio con una presentación de 250 $\mathrm{ml}$. Además, al obtener el costo real y el precio de venta es posible que pueda competir con las marcas Parmalat, La Perfecta y Eskimo.

\section{BIBLIOGRAFÍA}

Artica Mallqui, L. (2014). Métodos para el análisis físicoquímico de la leche y derivado lácteos. Huancayo, Perú: Editorial@Libros y editoriales, TEIA.

Di Rienzo J.A., C. F. (2016). InfoStat. Córdoba, Argentina: Universidad Nacional de Córdoba.

Mlranda, O., Fonseca, P. L., Ponce, I., \& Cedeño, C. (2014). Elaboración de una bebida fermentada a partir del suero de leche que incorpora Lactobacillus Acidophilus y Streptococcus Thermophilus. Revista Cubana de Alimentación y Nutrición, 7-16.

El Higo Revista Científica / Volumen 09.No.01, pp. 59-68 / Diciembre 2019 
Montesdeoca, R., Benítez, I., \& Guevara Raúl, G. G. (2017). Procedimiento para la producción de una bebida láctea fermentada utilizando lactosuero. Santiago: Revista chilena de nutrición.

Murphy, R. M. (2007). Introducción a los procesos químicos. En R. M. Murphy, Introducción a los procesos químicos (pág. 170). McGraw-Hill Interamericana.

Negri, L. (2017). http://www.aprocal.com.ar/wp-content/uploads/pH-y-acidez-en-leche2.org. Obtenido de El pH y acidez de la leche: http://www.aprocal.com.ar/wpcontent/uploads/pH-y-acidez-en-leche2.org

Parra Huertas, R. A. (2009). Lactosuero: Importancia en la industria de alimentos. Medellín: Universidad Pedagógica y Tecnológica de Colombia.

Román, J. H. (2005). Elaboración de un balance de masa para yogur en la planta de lácteos El Zamorano. Honduras: ZAMORANO.

Watts, B., G.L, Y., \& Jeffrey, L. (1992). Métodos sensoriales básicos para la evaluación de alimentos. Ontario, Canadá: Centro Internacional de Investigaciones para el Desarrollo.

\section{SEMBLANZA DE AUTORES}
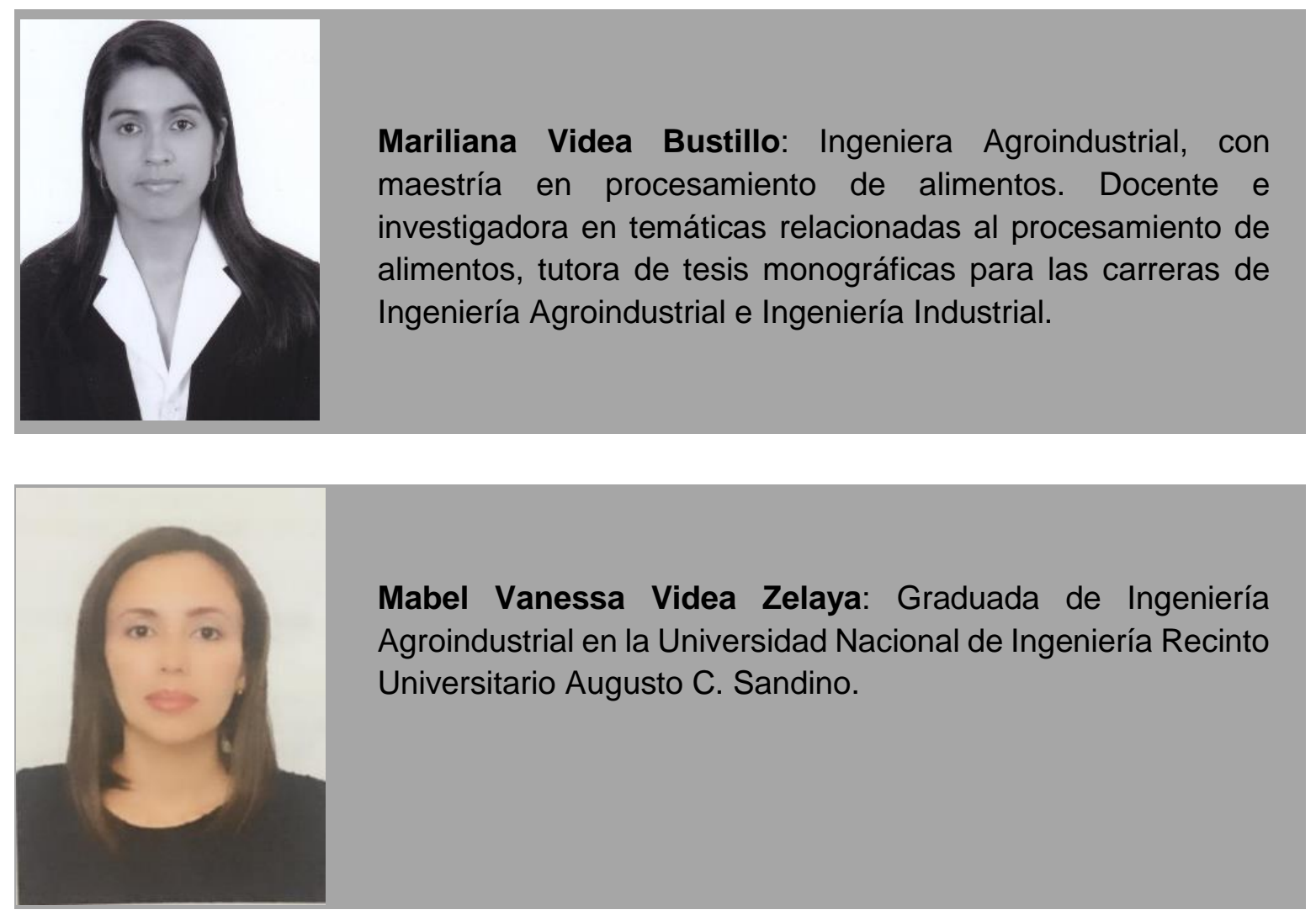

El Higo Revista Científica / Volumen 09.No.01, pp. 59-68 / Diciembre 2019 